\title{
RETRACTED ARTICLE: Internet of Things Based Intelligent Transportation of Food Products During COVID
}

\author{
Ala Saleh Alluhaidan ${ }^{1} \cdot$ Marwan Saleh Alluhaidan $^{2} \cdot$ Shakila Basheer $^{1}$
}

Accepted: 4 August 2021 / Published online: 21 August 2021

(C) The Author(s), under exclusive licence to Springer Science+Business Media, LLC, part of Springer Nature 2021

The Editor-in-Chief and the publisher have retracted this article. The article was submitted to be part of a guest-edited issue. An investigation by the publisher found a number of articles, including this one, with a number of concerns, including but not limited to compromised editorial handling and peer review process, inappropriate or irrelevant references or not being in scope of the journal or guest-edited issue. Based on the investigation's findings the Editor-in-Chief therefore no longer has confidence in the results and conclusions of this article. Shakila Basheer and Ala Saleh Alluhaidan have not explicitly stated whether they agree or disagree with this retraction. Marwan Saleh Alluhaidan has not responded to correspondence regarding this retraction.

The online version of this article contains the full text of the retracted article as Supplementary Information.

Supplementary Information The online version contains supplementary material available at https://doi. org/10.1007/s11277-021-08777-6.

Publisher's Note Springer Nature remains neutral with regard to jurisdictional claims in published maps and institutional affiliations.

Springer Nature or its licensor (e.g. a society or other partner) holds exclusive rights to this article under a publishing agreement with the author(s) or other rightsholder(s); author self-archiving of the accepted manuscript version of this article is solely governed by the terms of such publishing agreement and applicable law.

Shakila Basheer

sbbasheer@pnu.edu.sa

Ala Saleh Alluhaidan

ASALIuhaidan@pnu.edu.sa

Marwan Saleh Alluhaidan

Marwan1_2000@hotmail.com

1 Department of Information Systems, College of Computer and Information Science, Princess

Nourah Bint Abdulrahman University, Riyadh, Saudi Arabia

2 Ministry of Defense, Riyadh, Saudi Arabia 